\title{
Cohomology groups for projection tilings of codimension 2
}

\author{
Franz Gähler ${ }^{a}$, Johannes Kellendonk ${ }^{b}$ \\ ${ }^{a}$ Institut für Theoretische und Angewandte Physik, Universität Stuttgart, D-70550 Stuttgart
}

${ }^{b}$ Fachbereich Mathematik, MA 7-2, Technische Universität Berlin, D-10623 Berlin

30.8 .99

$\overrightarrow{\mathrm{A}}$ bstract

The gap-labelling group, which provides the set of possible values of the integrated density of states on gaps in the spectrum of a Hamiltonian describing particles in a tiling, is frequently related to the cohomology of the tiling. We present explicit results for the cohomology of many well-known tilings obtained from the cut and projection method with codimension 2, including the (generalized) Penrose tilings, the Tübingen-Triangle-Tiling, the Ammann-Beenker tiling, and the Socolar tiling.

Keywords: Quasicrystals, tilings, cohomology groups

\section{Introduction}

This article is about topological invariants for tilings, specifically for projection tilings, which play a major role in the description of quasicrystals. These invariants are cohomology groups of groupoids. The latter arise in the context of tilings since the motions from tile to tile do in general not form a group but rather a groupoid. For projection tilings, which are obtained from a higher-dimensional periodic structure, matters simplify enormously and we have several isomorphic descriptions of this cohomology which we simply call the cohomology of the tiling. First, it is isomorphic to the Czech-cohomology of the local isomorphism class of the tiling (suitably topologized). Second, and more important from the computational point of view, it is isomorphic to the cohomology of the higher-dimensional periodicity-lattice with coefficents derived from the local isomorphism class. And third it is isomorphic to the $K$-groups of the $C^{*}$ algebra associated with the groupoid.

These invariants yield an important step towards the classification of tilings. As they are invariants of the tiling groupoid, they are the same for locally isomorphic tilings and even for tilings which are mutually locally derivable [1] or topological equivalent [2]. We mention these equivalence relations because the construction of a tiling from an atomic structure will certainly not be unique.

Furthermore, the invariants play a role for the labelling of the gaps in the spectrum of a Hamiltonian describing the particle motion in the tiling. The $C^{*}$ algebra of the tiling groupoid turns out to be the algebra of observables. The Hamiltonian thus belongs to it and also its spectral projections associated with the gaps, i.e. projections on (generalized) eigenstates of all energies up to the gap. The $K_{0}$-class of such a projection furnishes a label for the gap which is stable under perturbations. Furthermore, there is a trace on the $C^{*}$-algebra coinciding in the physically relevant representation with the trace per unit volume. It induces a homomorphism from the $K_{0}$-group of the algebra to $\mathbb{R}$ and the image of this homomorphism is the gap-labelling group. The values of the integrated density of states on gaps belong to that group. More about this can be found in $[3,4,5]$. Unfortunately, the abovementionned isomorphism between cohomology and $K$-groups is only abstractly known. It would be desirable to have an explicit formula for it, or even more to have a direct physical interpretation of the cohomology groups which would allow us to interprete the results obtained below in physical terms. 


\section{Canonical projection tilings}

The class of tilings to which the results of $[6,7]$ apply are obtained by cut and projection from higherdimensional periodic structures. For the cut and projection method we use the formulation of [8]. The space such a tiling covers, here denoted $E$, is a linear space (of dimension $d$ ) embedded in a higherdimensional periodic polyhedral complex and its tiles are $d$-faces of that complex projected onto $E$. But not all $d$-faces are projected to yield tiles, only those for which the dual of the face (the complex comes with a fixed dual complex) intersects $E$. Starting with these data, $E$ and the polyhedral complex, one obtains in this way a tiling $\mathcal{T}$ provided the complex is in a regular position relative to $E$. The local isomorphism class $M \mathcal{T}$ of $\mathcal{T}$ can be obtained by looking not only at one fixed position for the complex but at all its translates. This gives a whole set of tilings. If one neglected the subtleties which arise if the complex is not in a regular position one would obtain a parametrization of this set by the torus $\left(E^{\perp} \times E\right) / \Gamma$, where $E^{\perp}$ is a complementary subspace of $E$, and $\Gamma$ the periodicity lattice. But the singular positions are most important for the topology of $M \mathcal{T}$. Each singular position of the complex actually yields two or more tilings, so that one has to make an additional choice to obtain a tiling. Let $S \subset E^{\perp}$ be the set of singular positions (interpreting a position relative to $E$ as a vector in $\left.E^{\perp}\right) . M \mathcal{T}$ can be described as the quotient $\left(E_{c}^{\perp} \times E\right) / \Gamma$ where $E_{c}^{\perp}$ is a completion of $E^{\perp} \backslash S$ in some non-euclidian topology and there exists a continuous surjection $E_{c}^{\perp} \rightarrow E^{\perp}$ having only one preimage for each regular point but several preimages for singular points. A singular point has as many preimages as there are additional choices, and all these preimages are in different connected components of $E_{c}^{\perp}$ although they are limit points of $E^{\perp} \backslash S$. This still doesn't describe completely the topology of $E_{c}^{\perp}$, but it illuminates how the topological information is encoded in the set $S$. In all interesting cases, the set $S$ can be described as follows: there is a finite set $\left\{H_{\alpha}\right\}_{\alpha}$ of hyperplanes of $E^{\perp}$ such that $S$ is the union of all its translates under the natural action of $\Gamma$. We call these planes and their translates singular planes. The $H_{\alpha}$ are simply the planes which are generated by the boundary faces of the so-called acceptance domains. The canonical choice is to take for the acceptance domains the projections of the duals of the $d$-faces onto $E^{\perp}$ (along $E$ ). If the tiling is decorated there may be further singular planes, whose points correspond to tilings which may carry different decorations.

\section{Cohomology groups}

The first important quantity which governs the qualitative behaviour of the cohomology is the number $L$ of $\Gamma$-orbits of intersection points, as we call points which constitute the intersection of $d^{\perp}$ singular planes. $d^{\perp}$ is the dimension of $E^{\perp}$ and is called the codimension of the tiling. If $L$ is infinite then the cohomology is infinitely generated [6]. There are simple criteria under which this is the case.

Suppose now that $L$ is finite. It is a general result that the cohomology group $H^{k}(\mathcal{T})$ of the tiling $\mathcal{T}$ is non-trivial only if $0 \leq k \leq d$. For $d^{\perp}=1$, one obtains $H^{d-k}(\mathcal{T}) \cong \mathbb{Z}^{\left(\begin{array}{l}d+1 \\ k+1\end{array}\right)}$ if $k>1$ and $H^{d}(\mathcal{T})=\mathbb{Z}^{d+L}[6]$ This is quite a simple result, in particular as $L=1$ for the canonical choice of the acceptance domain (no decoration). For $d^{\perp}=2$ the result is more complicated. It depends also on $H_{\alpha}$ and its stabilizers $\Gamma^{\alpha}$. More precisely, it depends on the rank of $\Gamma^{\alpha}$, on the number $l_{\alpha}$ of $\Gamma^{\alpha}$-orbits of intersection points in $H_{\alpha}$, and on the rank $r_{k}$ of the module generated by all submodules $\Lambda_{k+1} \Gamma^{\alpha} \subset \Lambda_{k+1} \Gamma$ ( $\Lambda \Gamma$ is the exterior

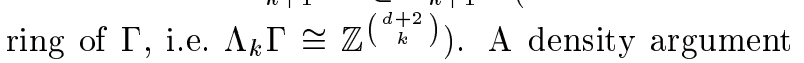
shows that in order to have finitely generated cohomology the ranks of all stabilizers $\Gamma^{\alpha}$ must be equal to $\frac{d+2}{2}$. If $f$ is the number of translation orbits of singular planes, one obtains [7] $H^{k}(\mathcal{T}) \cong \mathbb{Z}^{D_{k}}$, with

$$
\begin{aligned}
D_{d-k} & =\left(\begin{array}{c}
d+2 \\
k+2
\end{array}\right)-r_{k}-r_{k+1}+f\left(\frac{\frac{d+2}{2}}{k+1}\right), \quad k>0, \\
D_{d} & =\left(\begin{array}{c}
d+2 \\
2
\end{array}\right)-d-1-L-r_{1}+\sum_{\alpha \in I}\left(\frac{d}{2}+l_{\alpha}\right) .
\end{aligned}
$$

The cases of higher codimension become more involved, but there is a method to compute them [9].

\section{Results}

Using the formulae above, the cohomology has been computed for several well-known two-dimensional tilings with 5-, 8-, 10- and 12-fold symmetry, and for a four-dimensional tiling with 14-fold symmetry, all with $d^{\perp}=2$. The computations were done with a computer program which is a derivative of a program to compute Wyckoff positions of crystallographic space groups [10]. One basically had to enumerate the intersections of singular planes modulo lattice translations. For convenience, the singular planes and their intersections were further grouped into orbits under the point group of the lattice. The results are summarized in Table 1.

The octagonal and dodecagonal tilings in Table 1 are the usual Amman-Beenker and Socolar tilings, respectively $[11,12]$. The latter is mutually locally derivable with the shield tiling [13]. The matching rule decoration of these tilings induces a further set of singular planes [14], so that they are not locally derivable from their undecorated counterparts. The 
decagonal tilings are the TTT or Tübingen Triangle Tiling [1, 13], the Penrose tiling, and a generalized Penrose tiling with $\gamma \in \frac{1}{2}+\mathbb{Z}[\tau]$ (recall that the generalized Penrose tilings depend on an extra parameter $\gamma[15])$. We found that the cohomology distinguishes only two further classes of generalized Penrose tilings (with 5-fold symmetry), one for $\gamma \in \frac{1}{3}+\mathbb{Z}[\tau]$ or $\gamma \in \frac{2}{3}+\mathbb{Z}[\tau]$, and the generic case. The heptagonal tiling (which is actually even 14-fold symmetric) is four-dimensional, and has as acceptance domain a 14-gon, which is the convex hull of the projection of a generating 14-star of vectors of the lattice. Note that two-dimensional tilings with 7 - or 14-fold symmetry and polyhedral acceptance domain have all infinitely generated cohomology.

Acknowledgements. The second author is supported by the Sonderforschungsbereich 288 .

\section{References}

[1] M. Baake, M. Schlottmann, and P.D. Jarvis, Quasiperiodic tilings with tenfold symmetry and equivalence with respect to local derivability, in: J. Phys. A 24 (1991) 4637-4654.

[2] J. Kellendonk. Topological equivalence of tilings. J. Math. Phys. 38(4) (1997) 1823-1842.

[3] J. Bellissard, in: $K$-Theory of $C^{*}$-Algebras in Solid State Physics. In T.C. Dorlas, N.M. Hugenholtz, and M. Winnik (Eds.), Statistical Mechanics and Field Theory: Mathematical Aspects. Lecture Notes in Phys. 257, SpringerVerlag, 1986, 99-154.

[4] J. Bellissard. Gap labelling theorems for Schrödinger's operators. In M. Waldschmidt, P. Moussa, J.M. Luck, and C. Itzykson (Eds.), From Number Theory to Physics, Springer-Verlag, 1992, 538-630.

[5] J. Kellendonk. Non commutative geometry of tilings and gap labelling. Rev. Math. Phys. 7 (1995) 1133-1180.

[6] A.H. Forrest, J. Hunton, and J. Kellendonk. Projection quasicrystals II: Versus substitutions. SFB-preprint No. 396, 1999.

[7] A.H. Forrest, J. Hunton, and J. Kellendonk. Cohomology of canonical projection tilings. SFB-preprint No. 395, 1999.

[8] M. Schlottmann. Periodic and quasiperiodic Laguerre tilings. Int. J. Mod. Phys. B 7 (1993) 1351-1363.

[9] A.H. Forrest, J.R. Hunton, and J. Kellendonk. Projection quasicrystals III: Cohomology. in preparation.

[10] B. Eick, F. Gähler and W. Nickel. Computing Maximal Subgroups and Wyckoff Positions of Space Groups. Acta Cryst. A 53 (1997) 467-474.

[11] R. Ammann, B. Grünbaum and G.C. Shephard, Aperiodic Tiles. Discrete Comput. Geom. 8 (1992) 1-25.

[12] J.E.S. Socolar, Simple octagonal and dodecagonal quasicrystals. Phys. Rev. B 39 (1998) 10519-10551.

[13] R. Klitzing, M. Schlottmann and M. Baake. Perfet matching rules for undecorated triangular tilings with 10-, 12-, and 8-fold symmetry Int. J. Mod. Phys. B 7 (1993) 1455-1473.

[14] F. Gähler. Matching Rules for Quasicrystals: The Composition-Decomposition Method. J. Non-Cryst. Solids 153\&154 (1993) 160-164.
[15] M. Kleman and A. Pavlovitch. Generalized 2D Penrose tilings: structural properties. J. Phys. A: Math. Gen. 20 (1987) 687-702. 


\begin{tabular}{|l|l|l|l|c|c|c|c|c|c|c|}
\hline Tiling & $H^{0}$ & $H^{1}$ & $H^{2}$ & $H^{3}$ & $H^{4}$ & $L$ & $f$ & $l_{\alpha}$ & $r_{1}$ & $r_{2}$ \\
\hline \hline octagonal & $\mathbb{Z}$ & $\mathbb{Z}^{5}$ & $\mathbb{Z}^{9}$ & - & - & 3 & 4 & 2 & 3 & - \\
\hline octagonal (decorated) & $\mathbb{Z}$ & $\mathbb{Z}^{8}$ & $\mathbb{Z}^{23}$ & - & - & 8 & $4+4$ & 2,4 & 4 & - \\
\hline Penrose & $\mathbb{Z}$ & $\mathbb{Z}^{5}$ & $\mathbb{Z}^{8}$ & - & - & 1 & 5 & 1 & 4 & - \\
\hline decagonal TTT & $\mathbb{Z}$ & $\mathbb{Z}^{5}$ & $\mathbb{Z}^{24}$ & - & - & 5 & 5 & 5 & 4 & - \\
\hline $\begin{array}{l}\text { generalized Penrose } \\
\gamma \in \frac{1}{2}+\mathbb{Z}[\tau]\end{array}$ & $\mathbb{Z}$ & $\mathbb{Z}^{10}$ & $\mathbb{Z}^{34}$ & - & - & 15 & $5+5$ & 4,4 & 4 & - \\
\hline $\begin{array}{l}\text { generalized Penrose } \\
\gamma \in\left\{\frac{1}{3}, \frac{2}{3}\right\}+\mathbb{Z}[\tau]\end{array}$ & $\mathbb{Z}$ & $\mathbb{Z}^{10}$ & $\mathbb{Z}^{44}$ & - & - & 30 & $5+5$ & 6,7 & 4 & - \\
\hline $\begin{array}{l}\text { generalized Penrose } \\
\gamma \text { generic }\end{array}$ & $\mathbb{Z}$ & $\mathbb{Z}^{10}$ & $\mathbb{Z}^{34}$ & - & - & 40 & $5+5$ & 8,8 & 4 & - \\
\hline dodecagonal & $\mathbb{Z}$ & $\mathbb{Z}^{7}$ & $\mathbb{Z}^{28}$ & - & - & 14 & 6 & 6 & 3 & - \\
\hline dodecagonal (decorated) & $\mathbb{Z}$ & $\mathbb{Z}^{12}$ & $\mathbb{Z}^{59}$ & - & - & 24 & $6+6$ & 6,6 & 4 & - \\
\hline heptagonal $(d=4)$ & $\mathbb{Z}$ & $\mathbb{Z}^{6}$ & $\mathbb{Z}^{18}$ & $\mathbb{Z}^{25}$ & $\mathbb{Z}^{18}$ & 1 & 7 & 1 & 12 & 4 \\
\hline
\end{tabular}

Table 1: Cohomology groups of various codimension 2 tilings. For each tiling, also the quantities $L, f, l_{\alpha}$ and $r_{k}$ are given, which enter into the formulae for the cohomology groups. If the translation orbits of singular planes split into two orbits under the point group, their number $f$ is given as the sum of the lengths of these point group orbits. As $l_{\alpha}$ is constant on each point group orbit, it is listed only once per orbit, separated by a comma in the case of two orbits. 